\title{
Some Considerations on the Self-Motion Curves of a Planar 3R Manipulator
}

\author{
Jadran Lenarčič \\ "Jožef Stefan" Institute, Department of Automatics, Biocybernetics and Robotics, Ljubljana, Slovenia
}

\begin{abstract}
In this article, we evaluate the self-motion curves of a 3R planar manipulator possessing one-degree-ofredundancy, different link lengths and joint limits. We demonstrate that one or two self-motion curves correspond to a given combination of values of primary task coordinates. Because of the manipulator's mechanical restrictions, the self-motion can at one time be realized within one curve and the manipulator cannot switch to another curve without violating the constraints of the primary task. Partition of the self-motion domain into disconnected curves can disrupt the control process and thus decrease the ability of the manipulator to solve secondary tasks in the best possible way. We display and discuss the self-motion curves of this manipulator solving two different primary tasks.
\end{abstract}

Keywords: redundant robots, kinematic redundancy, self-motion curves, kinematic analysis, inverse kinematics.

\section{Introduction}

Multiple inverse kinematics solutions that correspond to some chosen values of task coordinates give to a robot manipulator higher kinematic flexibility as reported in [8]. Performance indices, such as manipulability or a norm of joint torques, can be varied by choosing different inverse kinematics solutions. In nonredundant manipulators, the number of inverse kinematics solutions is finite and we can only find a small discrete number of variations. In redundant manipulators, the number of inverse kinematics solutions is infinite (without regard to the degree of redundancy $D$ ) and an infinite number of variations may be obtained by moving the manipulator within a bounded domain of joint coordinates, as it was shown by $[3,4,6,12]$.
This motion of a redundant manipulator, which does not interfere with the primary task (usually but not exclusively positioning and orienting of the hand), is called the self-motion and is delimited in the joint space as a $D$-dimensional hyper-volume (curve or surface when $D$ is one or two, respectively). Hence, the self-motion is a resource (domain of joint coordinates) for solving secondary tasks, such as optimizing various performance indices, or avoiding obstacles and ill-conditioned configurations.

It was shown that when $D=1$ the manipulator's self-motion can be displayed by a curve in the space of joint coordinates. The self-motion curves were first characterized in [1]. They were later utilized to study the kinetic limitations of kinematically redundant manipulators in [11] and to produce fault tolerant operation in [10]. In order to produce fault-tolerant operation it would often be better to have the redundancy distributed over all joints. Hence, authors in [10] introduced the bounding box of the self-motion curves to measure the amount of redundancy, which takes into account the shape in addition to the size of the curve. The length of the operational self-motion curve was utilized as a measure to quantify the self-motion domain in $[7,9]$. A larger self-motion domain provides more freedom to solve secondary tasks but its size is not directly related to the range of values of a specific performance index whose optimization could be included in the secondary task. Nevertheless, an analysis of the manipulator's self-motion abilities provides very useful information for its design and especially for its control at the strategic level. 
The problem that is addressed in the present article is that the self-motion domain can in general be composed of a number of subspaces (disconnected curves when $D=1$ ) in the vector space of joint coordinates. For given values of primary task coordinates, the number of self-motion curves depends on the manipulator's mechanical constraints. Each curve corresponds to one group of inverse kinematics solutions where, for instance, one group of solutions may contain only positive values of a specific joint angle, while its counterpart may contain only negative values. The self-motion can only be realized within one group of solutions and the manipulator cannot switch to another group without violating the primary task constraints. The manipulator thus has at its disposal only part of the entire self-motion domain and may not be able to produce a desired solution to the secondary task although it is theoretically achievable. For example (see [7, 9]), a 4R anthropomorphic manipulator whose primary task is to position the wrist in space possesses eight disconnected self-motion curves throughout the entire reachable workspace. It means that this manipulator can simultaneously utilize only one eighth of its self-motion capacity without disturbing the imposed wrist position and, consequently, the solution of the secondary task depends also on the choice of the self-motion curve in which the manipulator operates.

In some cases, the number of self-motion curves changes as a function of primary task coordinates. This phenomenon introduces additional complexity and obstructs the image of the manipulator's self-motion ability. An example is the 3R planar manipulator discussed in this article (Fig. 1). Note that the 3R mechanism possesses a series of three parallel rotations. In some locations (singular configurations), one self-motion curve of length $s$ splits into two curves of lengths $s / 2$. The action of the manipulator in the neighborhood of these discontinuities can be troubled. For example, in two arbitrarily close primary task locations, one with single self-motion curve and one with a pair of curves, the control algorithm can produce two qualitatively different solutions to the secondary task if the "wrong" self-motion curve in the pair is chosen.

\section{Computational Background}

Determining the self-motion domain of redundant manipulators is usually very difficult and cannot be carried out by analytical calculations. Depending on the manipulator's structure and on the chosen primary task coordinates, we can use in some cases a semi-analytical technique [7], in which $D$ joint coordinates are numerically "swept" through different values, while the remaining coordinates are obtained via closedform inverse kinematics formulas. In general, however, we need to produce numerical solutions [2], which may introduce typical numerical problems, such as computational error, variable computational time, and number of iterations. The benefit of a numerical technique is in its universality. It may be applied to different manipulator structures and combinations of task coordinates.

Let $\mathbf{p}$ be the vector of $m$ primary task coordinates given as functions of $n$ joint angles q. When the manipulator is kinematically redundant, we have $n>m$. Vector $\mathbf{p}$ often includes a combination of constraints related to the manipulator's positions and/or orientations. In general, however, the list is not restricted to these two types of coordinates. In a differential form, vectors $\mathbf{q}$ and $\mathbf{p}$ are related by

$$
d \mathbf{p}=\mathbf{J} d \mathbf{q}
$$

Here, $\mathbf{J}$ denotes the $m \times n$ Jacobian matrix. One of possible reverse relationships is the following

$$
d \mathbf{q}=\mathbf{J}^{+} d \mathbf{p}
$$

where

$$
\mathbf{J}^{+}=\mathbf{J}^{T}\left(\mathbf{J J}^{T}\right)^{-1}
$$

is the unweighted pseudoinverse of matrix $\mathbf{J}$ whose dimension is $n \times m$ (see [5]). We mark the increment in joint angles that correspond to the self-motion by $d \mathbf{q}_{N}$. It is an element in the subspace of the vector space of vectors $d \mathbf{q}$. The fundamental property of the self-motion is that it does not produce any change in primary task coordinates $\mathbf{p}$. It means that for a non-zero increment $d \mathbf{q}_{N}$ the resultant increment $d \mathbf{p}=0$, so that (considering Eq. (1))

$$
\mathbf{J} d \mathbf{q}=0
$$


Vector $d \mathbf{q}_{N}$ can be computed as a linear transform of an arbitrary vector of joint increments $d \mathbf{q}$

$$
d \mathbf{q}_{N}=\mathbf{N} d \mathbf{q}
$$

where

$$
\mathbf{N}=\mathbf{I}-\mathbf{J}^{+} \mathbf{J}
$$

Matrix $\mathbf{N}$ is the $n \times n$ orthogonal complement of Jacobian matrix $\mathbf{J}$ since $\mathbf{J N}=0$ (combining Eqs. (4,5)). Matrix $\mathbf{N}$ is rank deficient and has the property of projecting an arbitrary vector (element of $n$-dimensional vector space) into the null space of matrix $\mathbf{J}$. Its rank defines the degree of manipulator's redundancy $D$

$$
D=\operatorname{rank}(\mathbf{N})=n-\operatorname{rank}(\mathbf{J})
$$

Outside kinematic singularities the degree of redundancy is $D=n-m$.

When $D=1$, the domain of the manipulator's self-motion is a (self-motion) curve in the space of joint coordinates. A procedure to obtain the self-motion curve can be based on a properly adapted method for computing the inverse kinematics. A more direct procedure, however, is to use Eq. (5). We initiate the computation in a desired point $\mathbf{q}^{(0)}$ on the self-motion curve and repeatedly add small joint increments $d \mathbf{q}_{N}^{(k)}$ as follows

$$
\mathbf{q}^{(k+1)}=\mathbf{q}^{(k)}+d \mathbf{q}_{N}^{(k)}, k=1,2, \ldots
$$

The length of each step on the curve is

$$
d \delta^{(k)}=\sqrt{\left(d \mathbf{q}_{N}^{(k)}\right)^{T} d \mathbf{q}_{N}^{(k)}}
$$

A weak point of the procedure is that it depends on arbitrarily chosen vector $d \mathbf{q}$ and on projection matrix $\mathbf{N}$, which may not ensure that the entire self-motion curve is acquired. An alternative is to consider that the vector space of $d \mathbf{q}_{N}$ is spanned by the $n$-dimensional eigenvectors of matrix $\mathbf{N N}^{T}$ corresponding to the $D$ non-zero eigenvalues (which are all real since $\mathbf{N N}^{T}$ is symmetric). When $D=1$, there is only one non-zero eigenvalue, so that the vector space of $d \mathbf{q}_{N}$ is characterized by one $n$-dimensional eigenvector e. Hence, we can introduce

$$
d \mathbf{q}_{N} \approx \gamma \mathbf{e}
$$

where $\gamma$ is a small scalar constant measured in radians, and $\mathbf{e}$ is assumed to be a unity vector.
The eigenvector $\mathbf{e}$ is identical to the eigenvector of matrix $\mathbf{J}^{T} \mathbf{J}$, which relates to the zero eigenvalue of $\mathbf{J}^{T} \mathbf{J}$. Now we can rewrite iteration (8) as follows

$$
\mathbf{q}^{(k+1)}=\mathbf{q}^{(k)}+\gamma \mathbf{e}^{(k)}, k=1,2, \ldots
$$

We choose $\gamma$ as a small positive constant (given in radians). Vector $\mathbf{e}^{(k)}$ is obtained from matrix $\left(\mathbf{J}^{(k)}\right)^{T} \mathbf{J}^{(k)}$ (or $\left.\left(\mathbf{N}^{(k)} \mathbf{N}^{(k)}\right)^{T}\right)$ which is a function of the actual values of joint angles $\mathbf{q}^{(k)}$. Note that a standard routine to compute the eigenvector may give solutions with opposite orientations. We select the direction of vector $\mathbf{e}^{(k)}$ by taking into account the orientation of its predecessor $\mathbf{e}^{(k-1)}$.

It must be pointed out that for some values of primary task coordinates the self-motion domain can be composed of two (or more) disconnected curves. The manipulator's self-motion can only be realized within one of these curves and the manipulator cannot switch to another curve unless the imposed primary task constraints are violated. The choice of the initial vector of joint angles $\mathbf{q}^{(0)}$ thus determines which of these curves will be computed by the algorithm. Clearly, the whole set of the selfmotion domain can be obtained by repeating the procedure with vectors $\mathbf{q}^{(0)}$ that correspond to different self-motion curves.

By the use of Eq. (11), the increment along the self-motion curve is constant in every $k=$ $1,2, \ldots$ and is approximately

$$
d \delta^{(k)} \approx \gamma
$$

The values of joint angles must be stored within an interval of $2 \pi$ in the following way

$$
\begin{aligned}
& \text { if } q_{i}^{(k)}>2 \pi \Rightarrow \tilde{q}_{i}^{(k)}-2 \pi \\
& \text { if } q_{i}^{(k)}<2 \pi \Rightarrow \tilde{q}_{i}^{(k)}+2 \pi
\end{aligned}
$$

$i=1,2, \ldots, n$, and then reset

$$
\mathbf{q}^{(k)}=\tilde{\mathbf{q}}^{(k)}
$$

$k=0,1, \ldots$ Note that the self-motion curve is a periodic function of joint angles. Hence, after a number of iterations, the computed vector $\mathbf{q}^{(k)}(11)$ returns to the neighborhood of the 
initial vector $\mathbf{q}^{(0)}$. The iterative procedure (11) is therefore stopped when both of the following inequalities are valid

$$
k>k_{0} \text { and }
$$

$$
\sqrt{\left(\mathbf{q}^{(k)}-\mathbf{q}^{(0)}\right)^{T}\left(\mathbf{q}^{(k)}-\mathbf{q}^{(0)}\right)}<\gamma k_{0}
$$

where $k_{0}$ is a chosen integer. The Euclidian norm in (17) represents the accumulated computational error along the self-motion curve. In the first approximation, this error depends linearly on the size of $\gamma$ and on the length of the curve. In the experiments that were performed with different redundant manipulators, this error never exceeded the value of $5 \gamma$. In practice, it is therefore appropriate to impose $k_{0} \geq 5$. The selection of $\gamma$ is a trade-off between the computational time and the accuracy. In a practical case, with an error smaller than $0.1 \%$, the execution time on a standard PC would be less than a few tens of a millisecond. Note that further numerical improvements could be introduced to enhance the accuracy. However, in applications, such as robot design, simulation, analysis and prediction of robot performance, an accuracy of a few percent does not play a decisive role.

The length of the self-motion curve is computed as the sum of all $d \delta^{(k)}, k=1,2, \ldots(11,12)$ as follows

$$
s=\sum_{k} \gamma
$$

When all joints are rotational, it is expressed in radians. It quantifies the domain (subspace in joint coordinates $\mathbf{q}$ ) of the self-motion of a redundant manipulator for a given vector of external coordinates $\mathbf{p}$.

We must be aware of the fact that in a singular configuration the degree of redundancy increases. The singularity point in the manipulator's configuration space is a cross of selfmotion curves. It must be pointed out, however, that the above numerical procedure is quite robust and is able to produce a continuous selfmotion curve, even when this passes very close to a kinematic singularity.

\section{Self-Motion Curves of a 3R Planar Manipulator}

In this paper we consider a 3R manipulator possessing three parallel rotations characterized by joint angles $\mathbf{q}=\left(q_{1}, q_{2}, q_{3}\right)^{T}$ and three links of lengths $d_{1}, d_{2}, d_{3}$ (as shown in Fig. 1). Let the list of task coordinates include the end effector position

$$
\begin{aligned}
p_{x}= & d_{1} \cos \left(q_{1}\right)+d_{2} \cos \left(q_{1}+q_{2}\right) \\
& +d_{3} \cos \left(q_{1}+q_{2}+q_{3}\right) \\
p_{y}= & d_{1} \sin \left(q_{1}\right)+d_{2} \sin \left(q_{1}+q_{2}\right) \\
& +d_{3} \sin \left(q_{1}+q_{2}+q_{3}\right)
\end{aligned}
$$

and orientation

$$
p_{\alpha}=q_{1}+q_{2}+q_{3}
$$

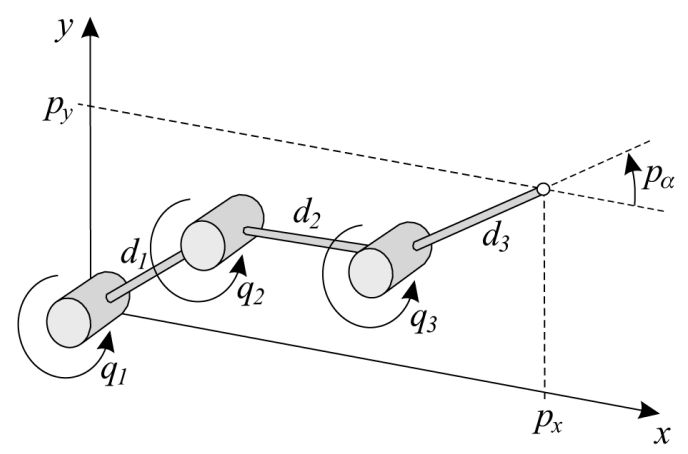

Fig. 1. 3R planar manipulator.

In the following examples, we assume that the joint angles are unlimited, the link lengths are $d_{1}=0.40 m, d_{2}=0.35 m, d_{3}=0.25 m$, and the step size is $\gamma=0.001$ radians. We consider two different pairs of primary task coordinates, in both cases $n=3, m=2$, and $D=1$. Note that the mechanism of the treated $3 \mathrm{R}$ manipulator is symmetric about the first joint axis pertinent to joint coordinate $q_{1}$. Therefore, there is no need to analyze the distribution of the self-motion domain $s$ in the whole $x y$ plane. A complete information is obtained only by studying $s$ along any radial axis perpendicular to the axis of $q_{1}$. In the following examples we plot $s$ along axis $x$. 


\subsection{Example with primary task coordinates $p_{x}$ and $p_{y}$}

In the first example, the primary task is to position the end effector at a given point $\mathbf{p}=$ $\left(p_{x}, p_{y}\right)^{T}$ with an unconstrained end effector orientation $p_{\alpha}$. The computed length $s(18)$ as a function of coordinate $p_{x}$ (while coordinate $\left.p_{y}=0\right)$ is shown in Fig. (2).

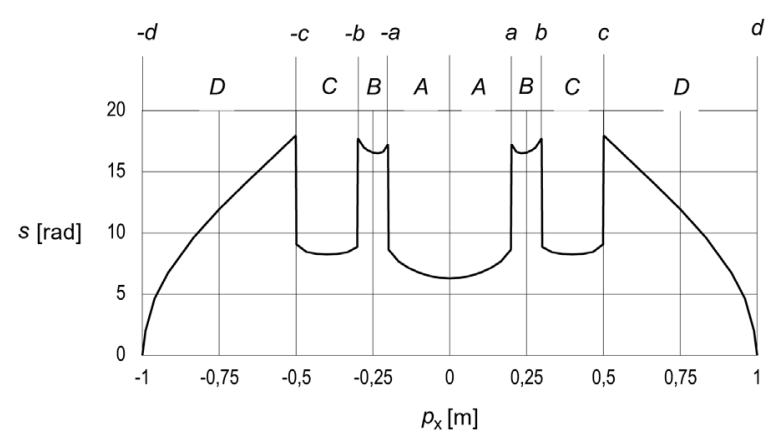

Fig. 2. Value of $s$ along $x$ - primary task coordinates $p_{x}, p_{y}$.

We can observe that there are four singular points along axis $x$ that separate the distribution of function $s$ into four distinctive regions. These points are denominated: $a=-d_{1}+d_{2}+d_{3}$, $b=d_{1}-d_{2}+d_{3}, c=d_{1}+d_{2}-d_{3}$ and $d=d_{1}+d_{2}+d_{3}$. Region $A$ are intervals $(0, a)$ and $(-a, 0)$, region $B$ are intervals $(a, b)$ and $(-b,-a)$, region $C$ are intervals $(b, c)$ and $(-c,-b)$, and region $D$ are intervals $(c, d)$ and $(-d,-c)$. The distinction between the regions is associated with the manipulator's configuration. In region $A$, the manipulator cannot (by its self-motion) switch from configurations with $q_{3}>0$ into configurations with $q_{3}<0$. In region $C$, the manipulator cannot switch from configurations with $q_{2}>0$ into configurations with $q_{2}<0$. The manipulator is free to pass from one group of configurations to another group in regions $B$ and $D$. Hence, in regions $A$ and $C$, the manipulator can (without any interruption) execute only one half of the self-motion. This property of the manipulator is the cause of the discontinuity of function $s$ in Fig. 2. We can thus conclude that the $3 \mathrm{R}$ manipulator is kinematically more flexible and contains more kinematic redundancy (relative to the above primary task) in regions $B$ and $D$.
The corresponding self-motion curves are plotted in Fig. 3. In regions $B$ and $D$, the manipulator produces one closed curve. In regions $A$ and $C$, two identical disconnected curves correspond to a given set of external coordinates but only one of them can be executed without interruptions. One curve in this pair represents one group of manipulator's configurations (in Fig. 3 only one curve of each pair is shown).
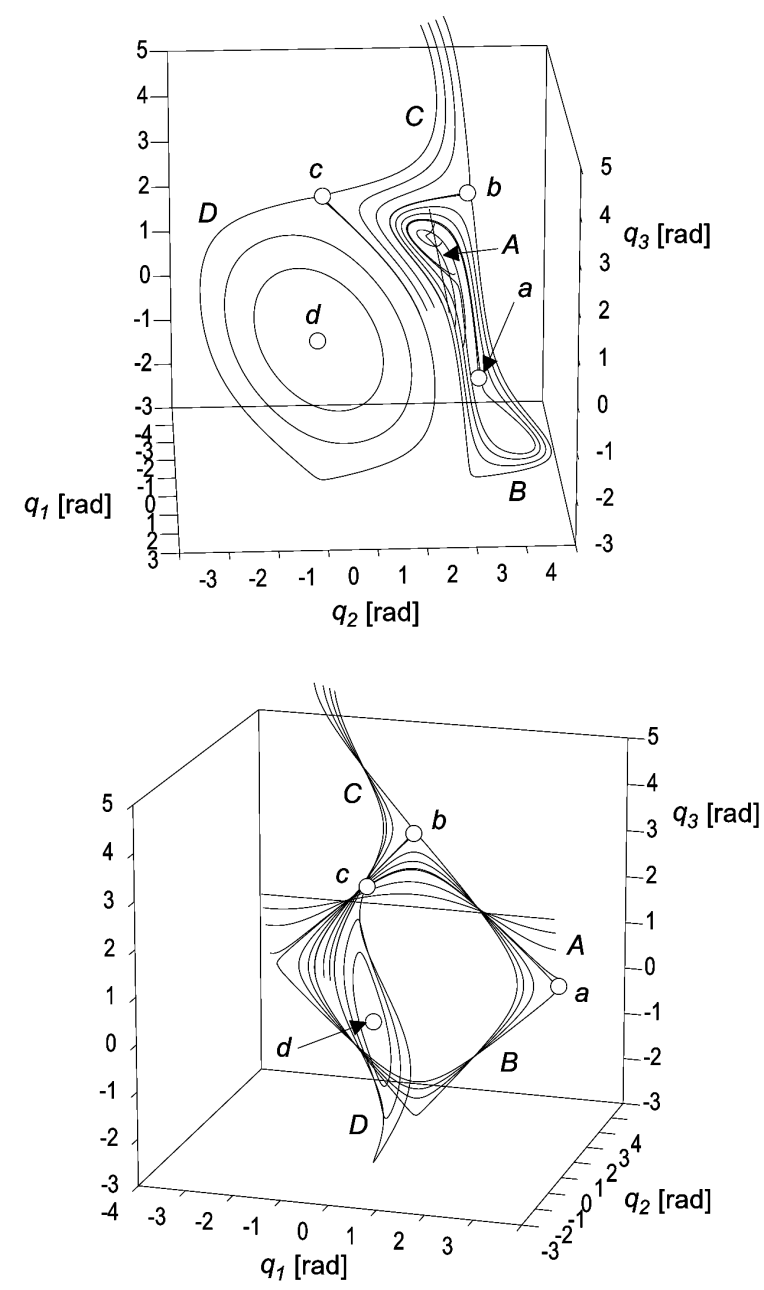

Fig. 3. Self-motion curves along $x$ - primary task coordinates $p_{x}, p_{y}$.

A specific case is when the manipulator possesses equal link lengths. In that case, two pairs of delimiting points collapse into two single points and the manipulator thus possesses only two different regions of the self-motion curves, one analogous to the above regions $A$, $C$ and the other analogous to the above regions $B, D$. 


\subsection{Example with primary task coordinates $p_{x}$ and $p_{\alpha}$}

In the second example, the primary task is to position and orient the end effector at the point $\mathbf{p}=\left(p_{x}, p_{\alpha}\right)^{T}$, while the end effector position $p_{y}$ is unconstrained. The computed length of the self-motion curve as a function of $p_{x}=\left(-d_{1}-d_{2}+d_{3}, d_{1}+d_{2}+d_{3}\right)$ and $p_{\alpha}$ is shown in Fig. 4. Here, the center of symmetry is at $p_{x}=d_{3}$.

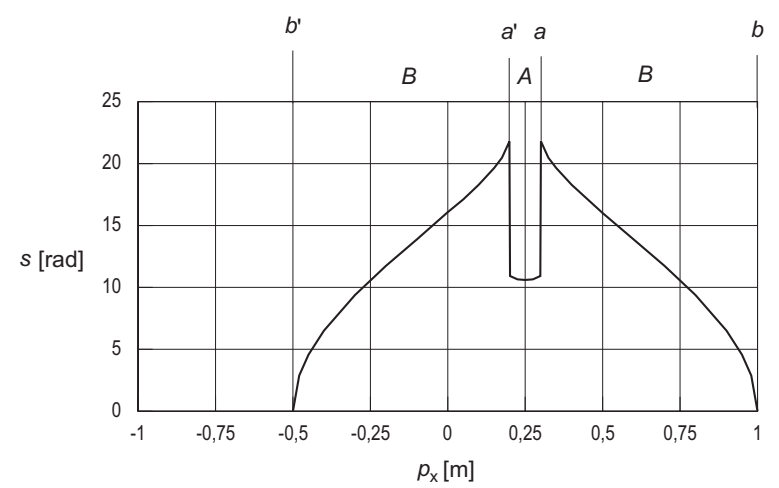

Fig. 4. Value of $s$ along $x$ - primary task coordinates $p_{x}, p_{\alpha}$.

We can see that there are two singular points along the $x$ axis that separate the distribution of function $s$ into two distinctive regions. One point is $a=d_{1}-d_{2}+d_{3}$ and its counterpart is $a^{\prime}=-d_{1}+d_{2}+d_{3}$, the second point $b=d_{1}+d_{2}+d_{3}$ and its counterpart is $b^{\prime}=$ $-d_{1}-d_{2}+d_{3}$. Region $A$ are intervals $\left(d_{3}, a\right)$ and $\left(a^{\prime}, d_{3}\right)$ region $B$ are intervals $(a, b)$ and $\left(b^{\prime}, a^{\prime}\right)$. The distinction between the regions in this example is associated with the reachability of the first two links. In region $A$, the manipulator's self-motion is restricted by an area that isn't reachable by the end point of the first two links and, therefore, it cannot switch from configurations with $q_{1}>0$ into configurations with $q_{1}<0$. The manipulator is able to cross from one group of configurations into another group in region $B$.

The corresponding self-motion curves are presented in Fig. 5. In regions $B$, the manipulator produces one closed curve for a given value of $p_{x}$. A pair of two identical disconnected curves corresponds to a given value of $p_{x}$ in region $A$ (only one of these is shown in Fig. 5). We can
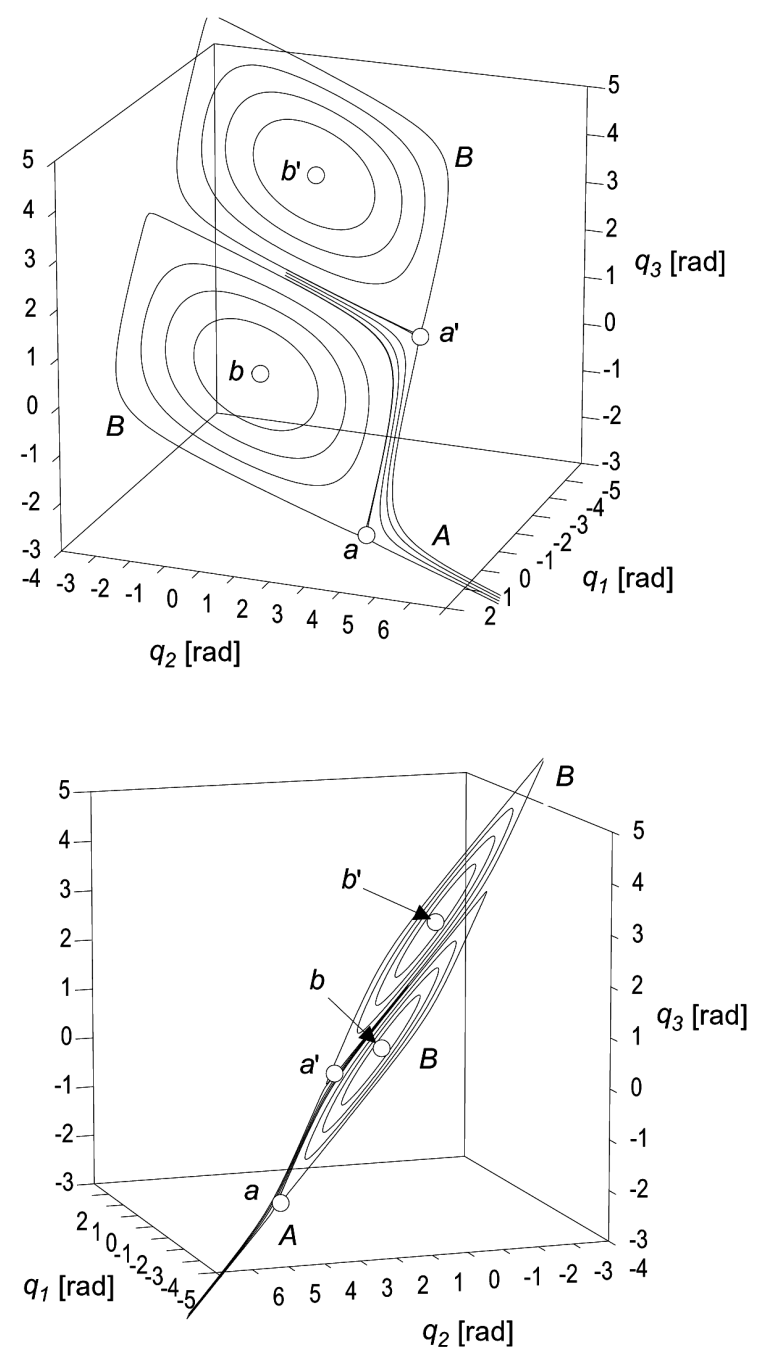

Fig. 5. Self-motion curves along $x$ - primary task coordinates $p_{x}, p_{\alpha}$.

observe that all self-motion curves in this example lie in one plane. It must be pointed out that a change in the size of the treated manipulator (as long as the relative sizes of its links remain intact) does not influence the self-motion curves and the corresponding lengths $s$.

\section{Conclusions}

In the experiments with the $3 \mathrm{R}$ manipulator (Fig. 1), we discovered regions in the joint space where the manipulator is unable to execute the complete range of its self-motion. These regions are associated with the inability of the manipulator to switch between different configurations (combinations of joint angles). The operational self-motion curve is thus 
shorter and the manipulator possesses less capacity to adapt to various secondary tasks.

The computational time of a self-motion curve based on the reported numerical procedure depends on its length, on the chosen resolution, and on the manipulator's structure. When the procedure is implemented on a standard personal computer, the computational time is in the range of a few tens of a millisecond. For this reason, the method may not be adequate for real-time control purposes. The main application is foreseen in the design, simulation and off-line programming of redundant robots.

An extension of the approach to manipulators with a combination of revolute and translational joints is in principle problematic since the length of the self-motion curves in these manipulators depends on the scaling factor between revolute and translational coordinates. On the other hand, an extension to revolute-joint manipulators possessing more degrees of redundancy is possible. The self-motion domain of these would be measured by the size of the selfmotion surface $(D=2)$, volume $(D=3)$, or hyper-volume $(D>3)$. The principal difficulty, however, is to ensure continuity in the searching direction since any linear combination of eigenvectors is utterly valid. Moreover, a representation of the wrinkled function $s$ in a general $D$-dimensional space may become quite obscure. Nevertheless, the limitation on manipulators with $D=1$ doesn't seem very impractical to us since many redundant manipulators are of this kind.

\section{References}

[1] J.W. BURDICK, On the inverse kinematics of redundant manipulators: characterization of the selfmotion manifolds. Presented at the Proceedings of the IEEE Conf. on Robotics and Automation, (1989) Scottsdale, USA.

[2] J.A. Euler, R.V. Dubey, S.M. BABCock, Selfmotion determination based on actuator velocity bounds for redundant manipulators., J. Robotics Systems, 6 (1989), 417-425.

[3] J. Hollerbach, Optimum kinematic design for a seven degree of freedom manipulator. Presented at the Proceedings of the 2nd Int. Symp. on Robotics Res., (1985) pp. 215-222, MIT Press.

[4] C.A. Klein, B.E. Blaho, Dexterity measures for the design and control of kinematically redundant manipulators. Int. J. Robotics Res., 6 (1987), 72-83.
[5] C.A. KLEIN, C.H. HuANG, Review of pseudoinverse control for use with kinematically redundant manipulators. IEEE Trans. on Syst., Man, and Cybern., 13 (1983), 245-250.

[6] K.P. LAM, Criteria optimization using self-motion of a 3-R redundant robot. J. Robotic Systems, 6 (1989), 254-267.

[7] J. LENARČIČ, The kinematic flexibility of articulated arms containing one degree of redundancy. Presented at the Proceedings of the 8th Int. Conf. on Advanced Robotics, (1997) Monterey, USA.

[8] J. LenARČIČ, U. Stanič, P. OBlaK, Study of kinematic flexibility of standard welding robots. Presented at the Proceedings of the 23rd Int. Symp. on Ind. Robots, (1992) Barcelona, Spain, pp. 277-282.

[9] J. LENARČIČ, The self-motion of an anthropomorphic manipulator. in Advances in Robot Kinematics: Analysis and Control (J. LENARČIČ, M.L. HUSTY, Eds.), (1998) pp. 571-578, Kluwer Academic Publishers, Dordrecht.

[10] C.L. LEWIS, A.A. MACIEJEWSKI, Fault-tolerant operation of kinematically redundant manipulators for locked joint failures. IEEE Trans. on Robotics and Automat. 13 (1997), 622-629.

[11] A.A. MACIEJEWSKI, Kinetic Limitations on the use of redundancy in robotic manipulators. IEEE Trans. on Robotics and Automat., 7 (1991), 205-210.

[12] K.J. WALDRON, J. REIDY, A study of a kinematically redundant manipulator structure. Presented at the Proceedings of the IEEE Conf. On Robotics and Automation, (1986) USA, pp. 1-8.

Received: January, 2001 Accepted: February, 2002

Contact address:
Jadran Lenarčič
"Jožef Stefan" Institute
Department of Automatics, Biocybernetics and Robotics
1000 Ljubljana, Slovenia
Phone: 386-1-477-33-78
Fax: 386-1-423-22-09
e-mail: jadran.lenarcic@ijs.si

JADRAN LENARČIČ graduated from the University of Ljubljana (Slovenia) in electrical engineering in 1979. He obtained M.Sc. and Ph.D degrees at the same university in 1981 and 1986, respectively. Since 1979 he has been with the "Jožef Stefan" Institute, where he is currently Head of the Department of Automatics, Biocybernetics and Robotics. His main research interests are in robot kinematics, humanoid robots and human biomechanics. He is co-editor of a series of books entitled Advances in Robot Kinematics published by Kluwer Academic Publishers. He was invited lecturer and visiting professor at universities all over the world. In 1999, he was elected a Regular Member of the Slovenian Academy of Engineering Sciences. 